\title{
Date of First Enrollment into Study
}

National Cancer Institute

\section{Source}

National Cancer Institute. Date of First Enrollment into Study. NCI Thesaurus. Code C139171.

The calendar date on which the first subject was enrolled into a study or trial. 